\title{
The Effect of Resveratrol on Bone Status in Rats with Bile Duct Obstruction
}

\author{
Helena Zivna ${ }^{1}$, Stanislav Micuda ${ }^{2}$, Eva Brcakova-Dolezelova ${ }^{2}$, Pavel Zivny ${ }^{3}$ \\ ${ }^{1}$ Radioisotope Laboratories and Vivarium, Medical Faculty, Charles University, Hradec Kralove, Czech Republic \\ ${ }^{2}$ Department of Pharmacology, Medical Faculty, Charles University, Hradec Kralove, Czech Republic \\ ${ }^{3}$ Institute of Clinical Biochemistry and Diagnostics, Medical Faculty, Charles University and University Hospital, Hradec Kralove, \\ Czech Republic \\ Email: zivna@1fhk.cuni.cz
}

Received November 23, 2012; revised December 25, 2012; accepted January 26, 2013

\begin{abstract}
Objective: We studied the influence of bile duct obstruction (BDO) after 28 days on bone metabolism status and their modification by resveratrol in male Wistar rats. Methods: The rats were divided into 3 groups: Sham group with laparotomy and vehiculum application, group BDO-bile duct obstruction and vehiculum application, and group R$\mathrm{BDO}$ - bile duct obstruction and resveratrol application $(10 \mathrm{mg} / \mathrm{kg}$ dose of resveratrol orally once daily). The bone mineral density (BMD; $\mathrm{g} / \mathrm{cm}^{2}$ ) and body composition were measured by dual energy X-ray absorptiometry. The physiccal strenght of femur was examined by controlled break biomechanical testing. The osteocalcin, procollagen type I $\mathrm{N}$-terminal propeptide (PINP) and carboxy-terminal collagen crosslinks (CTX) were analysed by EIA in the bone tissue homogenate. Results: The total BMD was lower in group BDO $(0.176 \pm 0.005 ; \mathrm{p}=0.01)$ and R-BDO $(0.181 \pm 0.004 ; \mathrm{p}$ $=0.052)$ vs Sham $(0.209 \pm 0.003)$. BMD of femur diaphysis in BDO group decreased significantly $(p=0.01)$ but not in R-BDO animals $(\mathrm{p}=0.052)$. Body fat $(\mathrm{g}$, median) was lower in BDO (19.0) and R-BDO (26.0) than in Sham (35.0). The force needed for fracture of femurs $(\mathrm{N})$ significantly decreased in BDO $(154 \pm 6)$ and R-BDO $(151 \pm 13)$ vs Sham group (208 \pm 7$)$. The force needed for neck fracture decreased in BDO $(105 \pm 3)$ and R-BDO $(115 \pm 8)$ vs Sham $(135 \pm$ 9). The osteocalcin decrease in group R-BDO $(2.9 \pm 0.1)$ vs Sham $(3.7 \pm 0.4)$. Higher PINP/CTX were in BDO $(20 \pm$ $5 / 0.49 \pm 0.08)$ and R-BDO $(30 \pm 4 / 0.55 \pm 0.07)$ vs Sham $(16 \pm 4 / 0.48 \pm 0.11)$. Conclusions: Our findings suggest that subchronic bile duct obstruction decreased body fat quantity and BMD with decrease of bone rigidity. Resveratrol showed protective effect on liver injury and consequently positive influence on bone tissue.
\end{abstract}

Keywords: Bone Metabolism; Rat; Resveratrol; Bile Duct Obstruction

\section{Introduction}

The osteoporosis has been recognized among others as a complication of chronic liver diseases such as haemochromatosis, alcoholic liver disease and particularly cholestatic disorders, namely primary biliary cirrhosis and primary sclerosing cholangitis. These patients have an increased risk of spontaneous or low-trauma fractures leading to significant morbidity, deterioration of quality of life, and even mortality [1,2]. The intesity of bone impairment which typically proceeds as "low-turnover" osteoporosis and related complications in these patients are proportional to the liver damage severity [3]. Although the underlying mechanisms are not completely understood, an imbalance in vitamine D turnover, calcium absorption or humoral signals such as IGF-1 deficiency or hyperbilirubinemia seems to be involved [4]. Advantageous in this situation is the possibility of using the reliable animal model based on the induction of long-term extrahepatic cholestasis in rats by bile duct obstruction or ligation. The animals develop bone disease which closely resembles human situation with low bone formation rate and low mass $[5,6]$ and can be used for studying therapeutic agents that may render protective effect in cholestatic liver diseases and associated bone changes $[7,8]$.

Resveratrol (3,5,4'-trihydroxy-trans-stilbene) is a stilbenoid (Figure 1), a type of natural phenol, and a phytoalexin produced naturally by several plants when under attack by pathogens such as bacteria or fungi [9]. Resveratrol, a powerful phytoestrogen, is contained in different plants, but especially in the skins of grapes. The molecule demonstrated wide variety of beneficial effects in cardiovascular diseases, cancers, diabetes, and neurodegenerative disorders [10]. With respect to liver diseases, although it also proved activity against NAFLD due to reduction of fatty acid availability [11], the major 
<smiles>Oc1ccc(/C=C/c2cc(O)cc(O)c2)cc1</smiles>

Figure 1. Structural formula of resveratrol.

effect is its strong anti-oxidative and anti-inflammatory activity which enables its hepatoprotective influence in different fibrotising long-term hepatic impairments including cholestatic one $[12,13]$. Improvement of the pathology is typically associated with the protection against hepatocellular injury as indicated by alleviated transaminase levels in serum and normalization of liver histology, mitigation of cholestasis with reduction of cumulating bile acids and bilirubin, and overall decrease in bioindicators of inflammation. Regarding bone tissue, resveratrol may inhibit the differentiation and bone resorbing activity of osteoclasts and promote the formation of osteoblasts from mesenchymal precursors in vitro [14]. On the other hand Shakibaei stated that resveratrol increased osteoclastic and decreased osteoblastic activities resulting in bone resorption and loss of bone mass [15]. The effect of the agent on the bone characteristics during cholestasis would be therefore interesting.

The osteocalcin (OC) is non-collagen protein, produced by active osteoblasts [16] and has been recognised as a bone-derived hormone to regulate energy metabolism [17]. Szalay reported decreased serum OC levels in patients with primary biliary cirrhosis and in those with chronic alcoholic liver disease [18]. Procollagen type I $\mathrm{N}$-terminal propeptide (PINP) is marker of collagen metabolism before its integration to bone matrix and reflects a bone formation. CTX-I (C-terminal crosslinking telopeptide of type I collagen) is released from C-terminal part of telopeptide of collagen I by proteolytic enzymes and its detection is a sensitive indicator of bone resorption [19].

We performed bile-duct obstruction (BDO) in rats to establish long-term cholestasis with bone impairment and evaluated the effect of resveratrol treatment. It is known that the risk of fracture strongly correlates with bone mineral density (BMD) and the best site for the hip fracture risk prediction is the measurement of the proximal femur BMD [1]. We therefore analyse the effects of $\mathrm{BDO}$ and the resveratrol on body composition, on the $\mathrm{BMD}$, and on the related biomechanical characteristics and metabolic markers of bone tissue.

\section{Experimental}

\subsection{Animals}

The effect of resveratrol on bone status in rats with bile duct obstruction

All animal procedures were performed in accordance with the guidelines set by the institutional Animal Use and Care Committee of Charles University, Prague, Faculty of Medicine in Hradec Kralove, Czech Republic. The protocol of the experiment was approved by the same committee. The adult albino Wistar rats (Biotest Ltd., Konarovice, CZ) were placed in plastic cages according to standard conditions (temperature $22^{\circ} \mathrm{C} \pm 2{ }^{\circ} \mathrm{C}$, 12 hours light/dark, air humidity $30 \%-70 \%$ ). The rats were divided into 3 groups (six - eight rats in each group). Initial body weight was $306 \pm 10 \mathrm{~g}$. All operations were performed in pentobarbital anesthesia $50 \mathrm{mg} / \mathrm{kg}$ body weight (Pentobarbital, Sigma-Aldrich, St. Louis, USA).

\subsection{Drugs}

Resveratrol (Sigma-Aldrich, Prague, CZ) was applied by gavage sond $10 \mathrm{mg} / \mathrm{kg}$ dose of resveratrol orally once daily in sterile water as vehiculum.

\subsection{Experimental Design}

Chronic cholestasis was induced by surgical bile duct obstruction (BDO) according to Brcakova et al. [20]. The cannula was inserted into the bile duct, closed and fixed subcutaneously. Obstruction lasted for 28 days. Control rats were either sham-operated, the bile duct was only manipulated and left unobstructed. 1) group: Sham: rats after laparotomy without bile duct obstruction and vehiculum application. 2) group: BDO: rats with bile duct obstruction and vehiculum application. 3) group: R-BDO: rats with bile duct obstruction and resveratrol application. The all rats were sacrificed after 28 days of experiment by exsanguination from abdominal aorta in pentobarbital anesthesia.

\subsection{Serum Biochemistry}

The blood samples were obtained during exsanguination. Serum aspartate-transaminase (AST; $\mu \mathrm{kat} / \mathrm{l}$ ), bile acids and total bilirubin concentrations $(\mu \mathrm{mol} / \mathrm{l})$ were estimated on automatic analyser Modular Roche.

\subsection{Bone Mineral Density Measurements}

The rat bone mineral density (BMD; g/ $\mathrm{cm}^{2}$; Figure 2) was measured by means of dual energy X-ray absorptiometry (DXA) on a Hologic Delphi A device (Hologic, Inc., Mass., USA) at the Osteocentre of the Faculty Hospital Hradec Kralove, Czech Republic. Before measurements, a tissue calibration scan was performed with the Hologic phantom for the small animal. BMD of the whole body, in the lumbar and tail vertebrae and in diaphysis of femurs, and total lean and fat were evaluated by computer using the appropriate software for small 


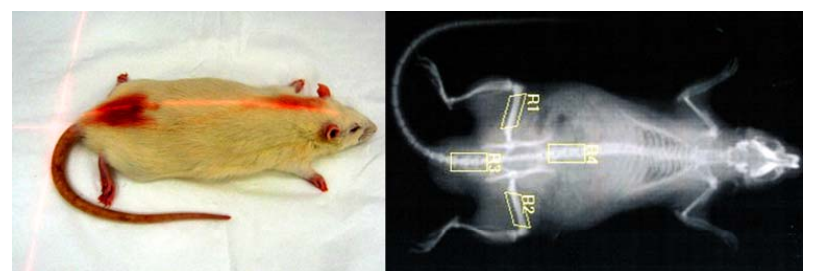

Figure 2. Measurement of BMD in rat.

animals (DXA; QDR-4500A Elite; Hologic, Inc., Waltham, USA).

\subsection{Bone Homogenate Analyses}

After animal sacrifice, both femurs and proximal tail vertebrae were carefully excised; after removal of all the surrounding skin, muscle and other soft tissue, and wrapped in saline-moistened gauze stored at $-80^{\circ} \mathrm{C}$ until required. Bone segments of the tail vertebra were obtained. Frozen samples $(150 \mathrm{mg})$ were minced in a phosphate buffer $(1.5 \mathrm{ml})$ and then disrupted and homogenized in a MagNA Lyser instrument (Roche Applied Science, Germany) during three rounds at 6500 rpm for $20 \mathrm{~s}$ with intermediate cooling $5 \mathrm{~min}$ in the MagNA Lyser Cooling Block. The tissue homogenate was centrifuged at $10,000 \mathrm{~g}$ at $4^{\circ} \mathrm{C}$ for $10 \mathrm{~min}$. The supernatant was separated and stored at $-80^{\circ} \mathrm{C}$. The bone markers osteocalcin, C-terminal part of telopeptide of collagen I and procollagen type I N-terminal propeptide concentrations in bone homogenate were assayed using a commercial rat ELISA kit manufactured by the firm Uscnlife Sciences \& Technology Co., Ltd., China (OC RatLaps $^{\text {TM }}$ EIA, $\mu \mathrm{g} / \mathrm{l}$; CTX-I, RatLaps ${ }^{\mathrm{TM}}$ EIA, $\mu \mathrm{g} / \mathrm{l}$; PINP, Rat/Mouse PINP EIA, $\mu \mathrm{g} / \mathrm{l})$.

\subsection{Bone Biomechanical Testing Procedure}

The both femurs were de-frosted in wet mull (saline solution). The mechanical properties of the femurs were performed and measured using special electro-mechanical custom-made testing machine (Kosek \& Trnecka, Hradec Kralove, CZ). A few hours before biomechemical testing, the femurs were thawed at room temperature and continuously moistened with isotonic saline solution. All specimens were tested at room temperature. The diaphysis midpoint was marked with a waterproof marking pen. The femur was placed on a holding device with the two support points located at a distance $18 \mathrm{~mm}$ apart. A small stabilizing preload to $10 \mathrm{~N}$ was applied at the medial surface of the diaphysis. A constant deformation rate of 6 $\mathrm{mm} / \mathrm{min}$ [21] until maximal load failure and bending stiffness was recorded. The three-point bending testing of the midfemur in the anteroposterior direction was used. Breaking strength (maximum load, N) was recorded (Figure 3).

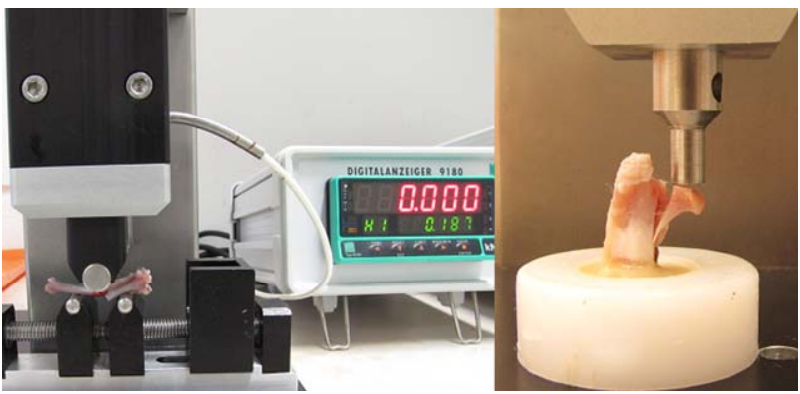

Figure 3. Example of three-point femur fracture with a measured pressure and femoral neck fracture.

The proximal part of femurs was used for compression test of femoral neck. The distal end of the broken femur was potted using self-curing adhesive methacrylate resin (Spofacryl-Spofa, Dental Ltd.; Jicin, CZ) into custommade casting containers. So then the bone was fixated. A vertical load conducted by a cylinder was applied to the top of the femoral head. A small stabilizing preload to 10 $\mathrm{N}$ was applied and used a constant speed of $6 \mathrm{~mm} / \mathrm{min}$ until fracture of the femoral neck. Breaking strength (maximum load, N) was determined (Figure 3). All bones were analyzed by the same operator.

\subsection{Statistical Analyses}

Holm-Sidak and Dunn's methods, One way ANOVA, pair t-test were performed by software "SigmaStat 3.1" Jandel Scientific ${ }^{\circledR}$, San Rafael, CA, USA. The data were expressed as mean $\pm \mathrm{SE}(\mathrm{p}<0.05)$ or median (percentil $25 \%-75 \%$ ). One sign represents statistical significance $p$ $<0.05$, two signs represent $\mathrm{p}<0.01$ and three signs represent $\mathrm{p}<0.001$.

\section{Results}

Serum biochemistry and body composition. The results of biochemical analysis indicates significant modulation of cholestatic liver injury by resveratrol-serum total bilirubin, bile acids and aspartate-transaminase which were markedly increased in untreated BDO rats were all mitigated by resveratrol therapy (Table 1).

In accordance, the body weight decreased mainly in BDO group, but not in group R-BDO. The amount of muscle and adipose tissues significantly declined in rats with BDO, but this decrease was prevented in rats after BDO with applied resveratrol (Table 2).

The bone mineral density 4 weeks after of BDO and $\mathrm{BDO}$ with resveratrol administration decreased in femur diaphysis of BDO group $(\mathrm{p}=0.01)$ but not in R-BDO animals $(p=0.052)$. The total BMD decreased statically significant in group BDL $(p=0.017)$ and $\mathrm{R}-\mathrm{BDL}(\mathrm{p}=$ 0.025 ; Figure 4). The same characters over columns labeled statistical significance between groups.

The bone fracture resistance of femurs decreased at 
Table 1. Serum biochemistry.

\begin{tabular}{cccc}
\hline & Bilirubin $(\mu \mathrm{mol} / \mathrm{l})$ & Bile acids $(\mu \mathrm{mol} / \mathrm{l})$ & AST $(\mu \mathrm{kat} / \mathrm{l})$ \\
\hline Sham & $2.3 \pm 0.5$ & $1.7 \pm 0.5$ & $3.1 \pm 1.5$ \\
& $69 \pm 26$ & $22 \pm 6$ & $43 \pm 28$ \\
BDO & $\mathrm{p}<0.001$ vs Sham & $\mathrm{p}<0.001$ vs Sham & $\mathrm{p}<0.01$ vs Sham \\
& $12 \pm 7$ & $9 \pm 7$ & $6.7 \pm 6.4$ \\
R-BDO & $\mathrm{p}<0.01$ vs Sham \\
& $\mathrm{p}<0.01$ vs BDO & $\mathrm{p}<0.001$ vs BDO & $\mathrm{p}<0.01$ vs BDO \\
\hline
\end{tabular}

Table 2. Body composition.

\begin{tabular}{|c|c|c|c|c|}
\hline & $\begin{array}{c}\text { Body } \\
\text { weight (g) }\end{array}$ & $\begin{array}{l}\text { Lean and } \\
\text { bone }(\mathrm{g})\end{array}$ & Fat (g) & Fat (\%) \\
\hline Sham & $392 \pm 9$ & $20.1 \pm 0.5$ & $\begin{array}{c}35 \\
(30-38)\end{array}$ & $\begin{array}{c}14 \\
(13-16)\end{array}$ \\
\hline BDO & $\begin{array}{l}342 \pm 10 \\
p=0.004 \\
\text { vs Sham }\end{array}$ & $\begin{array}{c}17.8 \pm 0.6 \\
\mathrm{p}=0.015 \mathrm{vs} \\
\text { Sham }\end{array}$ & $\begin{array}{c}19 \\
(15-21) \\
p<0.05 \text { vs } \\
\text { Sham }\end{array}$ & $\begin{array}{c}9.5 \\
(7-11) \\
p<0.05 \text { vs } \\
\text { Sham }\end{array}$ \\
\hline $\begin{array}{l}\text { R- } \\
\text { BDO }\end{array}$ & $\begin{array}{c}381 \pm 12 \\
\mathrm{p}=0.48 \mathrm{vs} \\
\text { Sham }\end{array}$ & $\begin{array}{c}18.8 \pm 0.8 \\
\mathrm{p}=0.198 \mathrm{vs} \\
\text { Sham }\end{array}$ & $\begin{array}{c}26 \\
(21-27)\end{array}$ & $\begin{array}{c}11 \\
(8-14)\end{array}$ \\
\hline
\end{tabular}

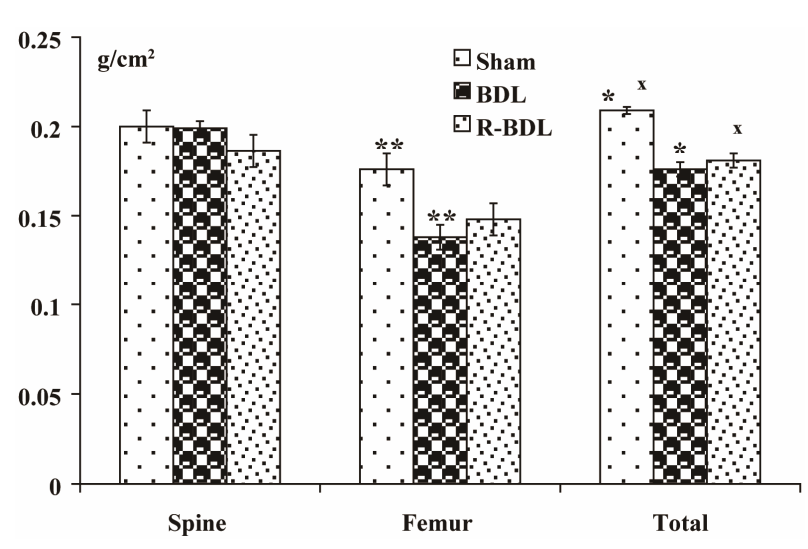

Figure 4. Bone mineral density.

group R-BDO ( $\mathrm{p}=0.004)$, but particularly at group $\mathrm{BDO}$ $(p<0.001)$. The fracture resistance of femur neck decreased in comparison with group Sham insignificantly at group R-BDO ( $\mathrm{p}=0.169)$, but significantly at group BDO ( $p=0.023$; Figure 5). The same characters over columns labeled statistical significance between groups.

Biochemistry of bone matrix turnover. The osteocalcin concentration decreased insignificantly in both groups with performed BDO. The concentration of CTX in these both experimental groups was not differed from group Sham. The significant enhancement of PINP concentration has been found at group R-BDO only ( $\mathrm{p}=0.038$; Table 3).

\section{Discussion}

In our study, the bile duct obstruction led to restraint of growth in rats, which was mitigated by resveratrol feeding.
Table 3. Biochemistry of bone markers.

\begin{tabular}{cccc}
\hline & Osteocalcin & PINP & CTX \\
\hline Sham & $3.71 \pm 0.47$ & $15.76 \pm 4.33$ & $0.483 \pm 0.112$ \\
BDO & $3.34 \pm 0.17$ & $20.07 \pm 5.43$ & $0.486 \pm 0.082$ \\
& $2.92 \pm 0.12$ & $30.16 \pm 4.20$ & $0.547 \pm 0.074$ \\
R-BDO & $\mathrm{p}=0.068$ vs Sham $\mathrm{p}=0.038$ vs Sham & $\mathrm{p}=0.834$ vs Sham \\
\hline
\end{tabular}

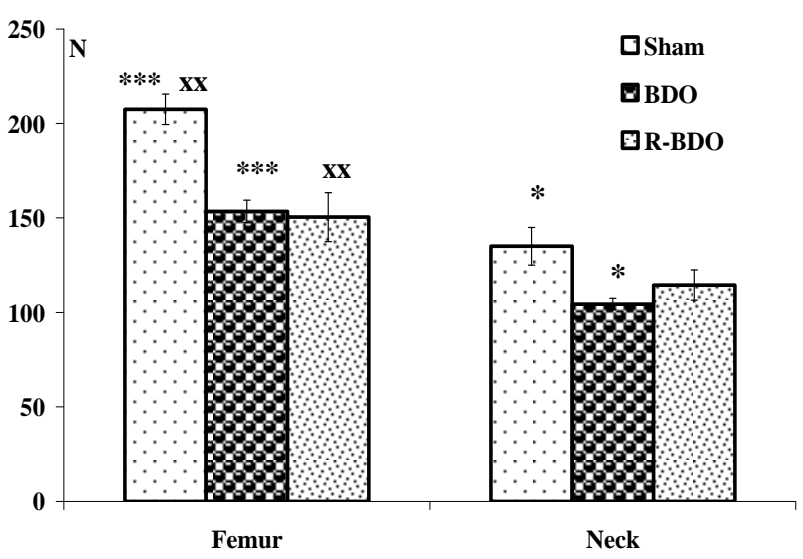

Figure 5. Bone fracture resistance.

Similarly, statistically significant reduction of muscle and adipose tissues occurred mainly in BDO rats, while resveratrol attenuated the impairment. This is an interesting finding because resveratrol feeding in healthy animals usually leads to body-fat lowering effect by reduction of fatty acid uptake and also de novo synthesis [22]. We therefore anticipate, that observed positive retaining of lean and fat mass in R-BDO animals is related to significant ability of resveratrol to attenuate the ongoing cholestatic liver injury. The involved mechanism is complex and it seems that with respect to lean and fat tissues it includes also preventive effect of resveratrol on bile duct ligation-mediated activation of hepatic stellate cells, in which BDO leads to reduced PPAR gamma expression [23] associated with activated leptin production, suppressed food intake, increased metabolic rate, and reduced fat depot size in untreated animals [24].

In accordance with results of other autors $[3,5,6]$ also our rats with $\mathrm{BDO}$ had reduction of bone mineral density after 4 weeks, and this was accompanied by diminution of bone strength and increased susceptibility for fracture especially in group BDO without resveratrol. When we compared experimental groups with and without resveratrol, slightly higher femoral BMD was found in rats with applied resveratrol. Previously, Mizutani indeed showed resveratrol ability of preventing decreases in femoral bone strength after ovariectomy, which was accompanied by elevation of femur hydroxyproline content [25]. On the other hand, Sehmisch referred that ovarectomised rats after 12 weeks feeding diet enriched by resveratrol did 
not have any changes in bone biomechanical strength or BMD [26]. We presume that absorption of vitamin D and calcium is impaired in situation of absolute cholestasis in BDO animals and resveratrol under these circumstances can not influence this mechanism.

The serum markers of the bone metabolism (osteocalcin, PINP and CTX-1) serve for evaluation of bone turnover. We therefore determined the local concentrations of these markers in the bone tissue homogenate to exclude other influences on the metabolism of the organism. The higher concentration of procollagen type I N-terminal propeptide (PINP) in group R-BDO indicates that resveratrol positively stimulate new formation of fibrous tissue locally in bone. This finding is similar to data presented by Singh who reported inhibition of the collagen type I, osteopontin, and bone sialoprotein formation by 2,3,7,8-tetrachlorodibenzo-p-dioxin from cigarette smoke, this effect was antagonized by resveratrol [27]. Moreover, resveratrol is also able to inhibit the receptor activator of nuclear factor- $\kappa \mathrm{B}$ ligand (RANKL)-mediated osteoclast differentiation, induces osteoclast apoptosis by inhibition of ROS production [28], activates osteoblastic activity in vitro by bone transcription of core binding factor $\alpha-1$ (Cbfa-1) [14], and reduce rosiglitazone-induced oxidative stress in osteoblast-like cells [29]. The resveratrol induces bone morphogenetic protein-2 through Src kinase-dependent estrogen receptor activation [30] and it could play a role in protecting against bone loss induced by estrogen deficiency [31], which arose after BDO.

In conclusion, the influence of resveratrol on bone is multifactorial; primarily changes in the bones themselves, demonstrated to rise of PINP. Secondarily the resveratrol has a positive effect on status of liver tissue and then tertiary on metabolic status, which leads consequence to improved condition for the bone tissue. The body weight also play an important role in regulating bone parameters in rats [32], as well as adequate amount of body fat. We assume that physical growth and formation of adipose tissue is slowed BDO and this situation was improved by application of resveratrol, and reflected in a better quality of bone tissue.

Interpretation of the results in rats requires only careful conclusions transfer into human medicine. Therefore we assume that application of resveratrol may be not limited in patients with osteoporosis during hepatic disease.

\section{Acknowledgements}

This study was supported by Prvouk P37/05; Ministry of Health, Czech Republic 00179906; SVV-2011-262902 and SVV-2012-264902.

\section{REFERENCES}

[1] G. Wariaghli, F. Allali, A. El Maghraoui and N. Hajjaj-
Hassouni, "Osteoporosis in Patients with Primary Biliary Cirrhosis," European Journal of Gastroenterology \& Hepatology, Vol. 22, No. 12, 2010, pp. 1397-1401.

[2] V. Goel and P. Kar, "Hepatic Osteodystrophy," Trop Gastroenterol, Vol. 31, No. 2, 2010, pp. 82-86.

[3] N. Guañabens, D. Cerdá, A. Monegal, F. Pons, L. Caballera, P. Peris and A. Parés, "Low Bone Mass and Severity of Cholestasis Affect Fracture Risk in Patients with Primary Biliary Cirrhosis," Gastroenterology, Vol. 138, No. 7, 2010, pp. 2348-2356. doi:10.1053/j.gastro.2010.02.016

[4] G. López-Larramona, A. J. Lucendo, S. González-Castillo and J. M. Tenias, "Hepatic Osteodystrophy: An Important Matter for Consideration in Chronic Liver Disease," World Journal of Hepatology, Vol. 3, No. 12, 2011, pp. 300-307. doi:10.4254/wjh.v3.i12.300

[5] F. A. Pereira, I. Facincani, V. Jorgetti, L. N. Ramalho, J. B. Volpon, L. M. Dos Reis and F. J. de Paula, "Etiopathogenesis of Hepatic Osteodystrophy in Wistar Rats with Cholestatic Liver Disease," Calcified Tissue International, Vol. 85, No. 1, 2009, pp. 75-83. doi:10.1007/s00223-009-9249-3

[6] M. Weinreb, R. D. Pollak and Z. Ackerman, "Experimental Cholestatic Liver Disease through Bile-Duct Ligation in Rats Results in Skeletal Fragility and Impaired Osteoblastogenesis," Journal of Hepatology, Vol. 40, No. 3, 2004, pp. 385-390. doi:10.1016/j.jhep.2003.11.032

[7] Z. Ackerman, M. Weinreb, G. Amir and R. D. Pollak, "Bone Mineral Metabolism and Histomorphometry in Rats with Cholestatic Liver Disease," Liver, Vol. 22, No. 2, 2002, pp. 166-172. doi:10.1046/j.0106-9543.2002.01566.x

[8] D. J. Leeming, D. V. Larsen, C. Zhang, Y. Hi, S. S. Veidal, R. H. Nielsen, K. Henriksen, Q. Zheng, V. Barkholt, B. J. Riis, I. Byrjalsen, P. Qvist and M. A. Karsdal, "Enzyme-Linked Immunosorbent Serum Assays (ELISAs) for Rat and Human N-Terminal Pro-Peptide of Collagen Type I (PINP)-Assessment of Corresponding Epitopes," Clinical Biochemistry, Vol. 43, No. 15, 2010, pp. 12491256. doi:10.1016/j.clinbiochem.2010.07.025

[9] F. Melchior and H. Kindl, "Grapevine Stilbene Synthase cDNA Only Slightly Differing from Chalcone Synthese cDNA Is Expressed in Escherichia Coli into a Catalytically Active Enzyme," FEBS Letters, Vol. 268, No. 1, 1990, pp. 17-20. doi:10.1016/0014-5793(90)80961-H

[10] W. Yu, Y. C. Fu and W. Wang, "Cellular and Molecular Effects of Resveratrol in Health and Disease," Journal of Cellular Biochemistry, Vol. 113, No. 3, 2012, pp. 752759. doi:10.1002/jcb. 23431

[11] S. Gómez-Zorita, A. Fernández-Quintela, M. T. Macarulla, L. Aguirre, E. Hájina, L. Bujana, F. Milagro, J. A. Martínez and M. P. Portillo, "Resveratrol Attenuates Steatosis in Obese Zucker Rats by Decreasing Fatty Acid Availability and Reducing Oxidative Stress," British Journal of Nutrition, Vol. 107, No. 2, 2012, pp. 202-210. doi:10.1017/S0007114511002753

[12] T. K. Lin, L. T. Huang, Y. H. Huang, M. M. Tiao, K. S. Tang and C. W. Liou, "The Effect of the Red Wine Polyphenol Resveratrol on a Rat Model of Biliary Obstructed Cholestasis: Involvement of Anti-Apoptotic Signalling, Mitochondrialbiogenesis and the Induction of Autophagy," 
Apoptosis, Vol. 17, No. 8, 2012, pp. 871-879. doi:10.1007/s10495-012-0732-3

[13] C. C. Chan, L. Y. Cheng, C. L. Lin, Y. H. Huang, H. C. Lin and F. Y. Lee, "The Protective Role of Natural Phytoalexin Resveratrol on Inflammation, Fibrosis and Regeneration in Cholestatic Liver Injury," Molecular Nutrition \& Food Research, Vol. 55, No. 12, 2011, pp. 1841-1849. doi:10.1002/mnfr.201100374

[14] K. Kupisiewicz, P. Boissy, B. M. Abdallah, F. D. Hansen, R. G. Erben, J. F. Savouret, K. Søe, T. L. Andersen, T. Plesner and J. M. Delaisse, "Potential of Resveratrol Analogues as Antagonists of Osteoclasts and Promoters of Osteoblasts," Calcified Tissue International, Vol. 87, No. 5, 2010, pp. 437-449. doi:10.1007/s00223-010-9399-3

[15] M. Shakibaei, C. Buhrmann and A. Mobasheri, "Resveratrol-Mediated SIRT-1 Interactions with p300 Modulate Receptor Activator of NF-Kappab Ligand (RANKL) Activation of NF-Kappab Signaling and Inhibit Osteoclastogenesis in Bone-Derived Cells," The Journal of Biological Chemistry, Vol. 26, No. 9, 2011, pp. 1173-1185.

[16] R. L. Duncan, K. A. Akanbi and M. C. Farach-Carson, "Calcium Signals and Calcium Channels in Osteoblastic Cells," Seminars in Nephrology, Vol. 18, No. 2, 1998, pp. 178-190.

[17] J. M. Fernández-Real and W. Ricart, "Osteocalcin: A new Link between Bone and Energy Metabolism. Some Evolutionary Clues," Current Opinion in Clinical Nutrition \& Metabolic Care, Vol. 14, No. 4, 2011, pp. 360-366. doi:10.1097/MCO.0b013e328346df4e

[18] F. Szalay, P. Lakatos, J. Németh, M. Abonyi, B. Büki, G. Tarján and I. Holló, "Decreased Serum Osteocalcin Level in Non-Alcoholic and Alcoholic Chronic Liver Diseases," Orvosi Hetilap, Vol. 132, No. 24, 1991, pp. 1301-1305.

[19] M. Herrmann, N. Umanskaya, B. Wildemann, G. Colaianni, T. Widmann, A. Zallone and W. Hermann, "Stimulation of Osteoblast Activity by Homocysteine," Journal of Cellular and Molecular Medicine, Vol. 12, No. 4, 2008, pp. 1205-1210. doi:10.1111/j.1582-4934.2008.00104.X

[20] E. Brcakova, L. Fuksa, J. Cermanova, G. Kolouchova, M. Hroch, P. Hirsova, J. Martinkova, F. Staud and S. Micuda, "Alteration of Methotrexate Biliary and Renal Elimination during Extrahepatic and Intrahepatic Cholestasis in Rats," Biological and Pharmaceutical Bulletin, Vol. 32, No. 12, 2009, pp. 1978-1985. doi:10.1248/bpb.32.1978

[21] C. H. Turner and D. B. Burr, "Basic Biomechanical Measurements of Bone: A Tutorial," Bone, Vol. 14, No. 4, 1993, pp. 595-608. doi:10.1016/8756-3282(93)90081-K

[22] G. Alberdi, V. M. Rodríguez, J. Miranda, M. T. Macarulla, N. Arias, C. Andrés-Lacueva and M. P. Portillo, "Changes in White Adipose Tissue Metabolism Induced by Resveratrol in Rats," Nutrition \& Metabolism, Vol. 8, No. 1, 2011, p. 29. doi:10.1186/1743-7075-8-29

[23] T. Miyahara, L. Schrum, R. Rippe, S. Xiong, H. F. Yee Jr., K. Motomura, F. A. Anania, T. M. Willson and H. Tsu- kamoto, "Peroxisome Proliferator-Activated Receptors and Hepatic Stellate Cell Activation," The Journal of Biological Chemistry, Vol. 275, No. 46, 2000, pp. 35715-35722. doi:10.1074/jbc.M006577200

[24] J. J. Potter, L. Womack, E. Mezey and F. A. Anania, "Transdifferentiation of Rat Hepatic Stellate Cells Results in Leptin Expression," Biochemical and Biophysical Research Communications, Vol. 244, No. 2, 1998, pp. 178-182. doi:10.1006/bbrc.1997.8193

[25] K. Mizutani, K. Ikeda, Y. Kawai and Y. Yamori, "Resveratrol Attenuates Ovariectomy-Induced Hypertension and Bone Loss in Stroke-Prone Spontaneously Hypertensive Rats," Journal of Nutritional Science and Vitaminology, Vol. 46, No. 2, 2000, pp. 78-83. doi:10.3177/jnsv.46.78

[26] S. Sehmisch, F. Hammer, J. Christoffel, D. SeidlovaWuttke, M. Tezval, W. Wuttke, K. M. Stuermer and E. K. Stuermer, "Comparison of the Phytohormones Genistein, Resveratrol and 8-Prenylnaringenin as Agents for Preventing Osteoporosis," Planta Medica, Vol. 74, No. 8, 2008, pp. 794-801. doi:10.1055/s-2008-1074550

[27] S. U. Singh, R. F. Casper, P. C. Fritz, B. Sukhu, B. Ganss, B. Girard Jr., J. F. Savouret and H. C. Tenenbaum, "Inhibition of Dioxin Effects on Bone Formation in Vitro by a Newly Described Aryl Hydrocarbon Receptor Antagonist, Resveratrol," Journal of Endocrinology, Vol. 167, No. 1, 2000, pp. 183-195. doi:10.1677/joe.0.1670183

[28] X. He, G. Andersson, U. Lindgren and Y. Li, "Resveratrol Prevents RANKL-Induced Osteoclast Differentiation of Murine Osteoclast Progenitor RAW 264.7 Cells through Inhibition of ROS Production," Biochemical and Biophysical Research Communications, Vol. 401, No. 3, 2010, pp. 356-362. doi:10.1016/j.bbrc.2010.09.053

[29] C. Bruedigam, M. Eijken, M. Koedam, H. Chiba and J. P. van Leeuwen, "Opposing Actions of Rosiglitazone and Resveratrol on Mineralization in Human Vascular Smooth Muscle Cells," Journal of Molecular and Cellular Cardiology, Vol. 51, No. 5, 2011, pp. 862-871. doi:10.1016/j.yjmcc.2011.07.020

[30] J. L. Su, C. Y. Yang, M. Zhao, M. L. Kuo and M. L. Yen, "Forkhead Proteins Are Critical for Bone Morphogenetic Protein-2 Regulation and Anti-Tumor Activity of Resveratrol," The Journal of Biological Chemistry, Vol. 282, No. 27, 2007, pp. 19385-19398. doi:10.1074/jbc.M702452200

[31] Z. P. Liu, W. X. Li, B. Yu, J. Huang, J. Sun, J. S. Huo and C. X. Liu, "Effects of Trans-Resveratrol from polygonum Cuspidatum on Bone Loss Using the Ovariectomized Rat Model," Journal of Medicinal Food, Vol. 8, No. 1, 2005, pp. 14-19. doi:10.1089/jmf.2005.8.14

[32] L. L. Chen, S. X. Wang, Y. Dai, P. Buckoreelall, P. Zhang, H. H. Zhang and W. Kong, "Effect of Catch-Up Growth by Various Dietary Patterns and Resveratrol Intervention on Bone Status," Experimental Biology and Medicine, Vol. 237, No. 3, 2012, pp. 297-304. doi:10.1258/ebm.2011.011296 